\title{
Botulinum toxin A treatment of overactive corrugator supercilii in thyroid eye disease
}

\author{
Jane M Olver
}

\begin{abstract}
Backgroundlaim-Patients with thyroid eye disease with upper eyelid retraction often develop overaction of the accessory muscles of eyelid closure, the glabellar muscles corrugator supercilii and procerus. The resultant glabellar furrowing (frown lines) contributes to the typical thyroid facies. The aim of this study was to evaluate the use of botulinum toxin $A$ reversible chemodenervation of the glabellar muscles as adjunctive treatment in the rehabilitation of patients with thyroid eye disease.
\end{abstract}

Methods-14 patients (13 females) ages 39-76 years (mean 52) with inactive thyroid eye disease and associated medial eyebrow ptosis and prominent glabellar frown lines were recruited. All patients had a history of upper eyelid retraction. Each patient was treated with a single botulinum toxin injection (Dysport $0.2 \mathrm{ml}$, 40 units) into each corrugator supercilii and sometimes procerus muscles as an outpatient procedure. The effectiveness and acceptability of the treatment was assessed clinically and from a patient questionnaire.

Results-The injections were tolerated by $13 / 14(93 \%)$ patients. There was resultant flattening of the glabellar region and improvement of medial eyebrow contour in all patients, with onset of paralysis within 1 week. All patients reported a subjective improvement in appearance. Side effects included one patient $(7 \%)$ with reversible partial ptosis. The beneficial effect lasted 4-6 months, with a gradual return of function. Repeat treatment was indicated where there was persistent upper eyelid retraction and protractor overaction.

Conclusion-Botulinum toxin A chemodenervation of the glabellar muscles in these patients was effective and acceptable. Chemodenervation should be considered in the rehabilitation of patients with thyroid eye disease where there is upper eyelid retraction and overacting protractors resulting in a thyroid frown. Once the eyelid retraction has been successfully treated by surgery, the need for further glabella muscle chemodenervation is considerably reduced.

Western Eye Hospital, Marylebone Road, London NW1

J M Olver

Accepted for publication 19 November 1997 appearance is physically and psychologically disabling. The frown is characterised by vertical glabellar furrowing and an altered eyebrow contour, with bunching up of the medial eyebrow, a medial brow ptosis, and often a deep horizontal line over the bridge of the nose. Some patients have referred to this appearance as an "angry scowl". These facial lines are the result of chronic contraction of corrugator supercilii and procerus muscles pulling on the skin, and differ from fine facial wrinkles which are largely due to dermal collagen age changes.

It is well recognised that flattening of the glabellar region occurs with botulinum toxin A treatment of blepharospasm ${ }^{2-4}$; this observation led to the specific treatment of glabellar hyperfunction lines with toxin. ${ }^{4-8}$

The aim of this study was to evaluate the use of botulinum toxin A chemodenervation of corrugator supercilii and procerus, as an adjunctive treatment in the rehabilitation of patients with thyroid eye disease with an angry looking frown.

\section{Anatomy ${ }^{9-13}$ \\ See Figure 1.}

The glabellar muscles, corrugator supercilii, and procerus are both accessory protractors of the upper lid, supplied by the facial nerve. Their action helps reduce glare in bright sunlight by drawing the medial end of the eyebrows medially and inferiorly, thus acting as accessory muscles of upper eyelid closure. They are also mimetic muscles which are typically contracted in anger or perplexity. They form part of the superficial musculoaponeurotic system (SMAS) of the upper face.

Corrugator supercilii is a small pyramidal muscle at the medial end of the eyebrow, in most part deep to the frontal part of occipitofrontalis and the orbicularis oculi. It originates from deep orbicularis oculi fibres, ${ }^{11}$ frontal bone, and frontal process of the maxilla at the superomedial orbital rim. ${ }^{9-11}$ From the medial end of the eyebrow the fibres pass laterally and slightly upwards, through the frontalisorbicularis interdigitation, to insert into the dermis of the middle third of the eyebrow above the supraorbital margin. The supratrochlea nerve and supraorbital nerve penetrate the corrugator muscle before passing superficially. The action of paired corrugator supercilii muscles is to draw the eyebrows downwards (inferiorly) and medially, producing vertical glabellar furrows. ${ }^{1011} 13$ Contraction occurs particularly in bright sunlight where it is acting as an accessory protractor to orbicularis oculi. 


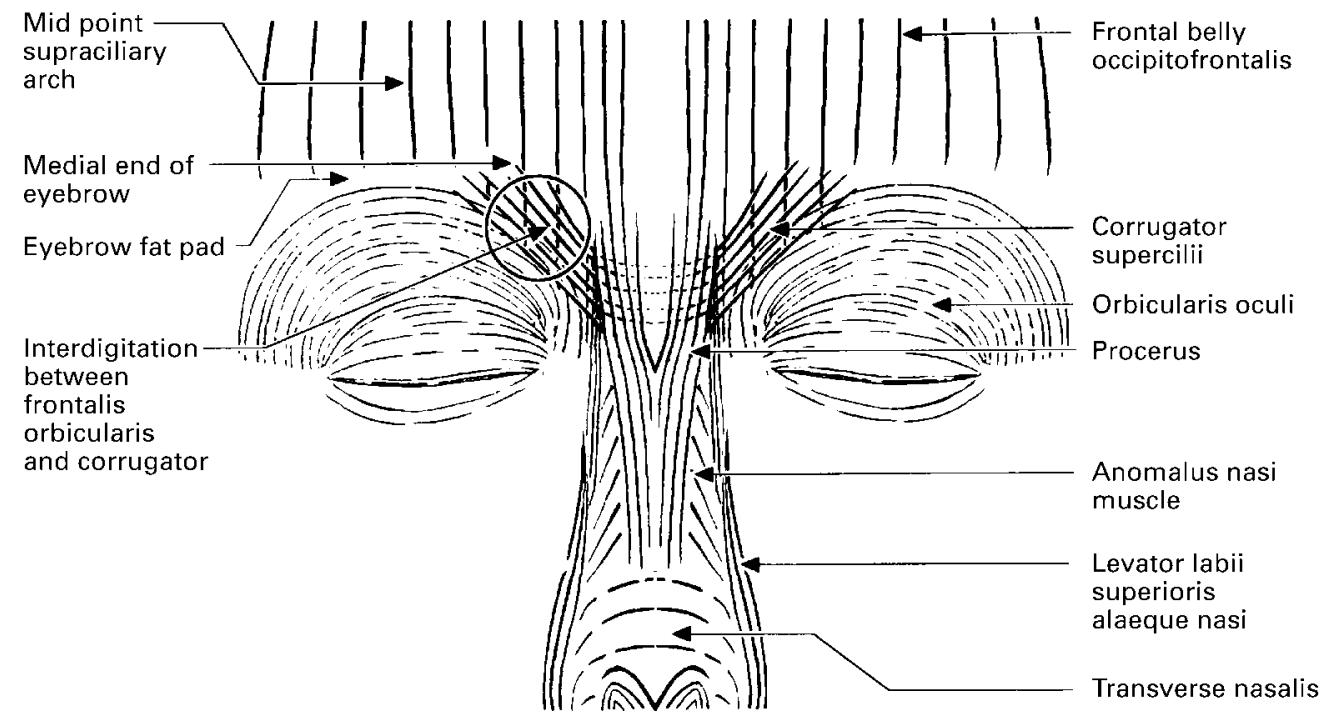

Figure 1 The corrugator supercilii muscles interdigitate with both frontalis and orbicularis at the medial end of the eyebrow.
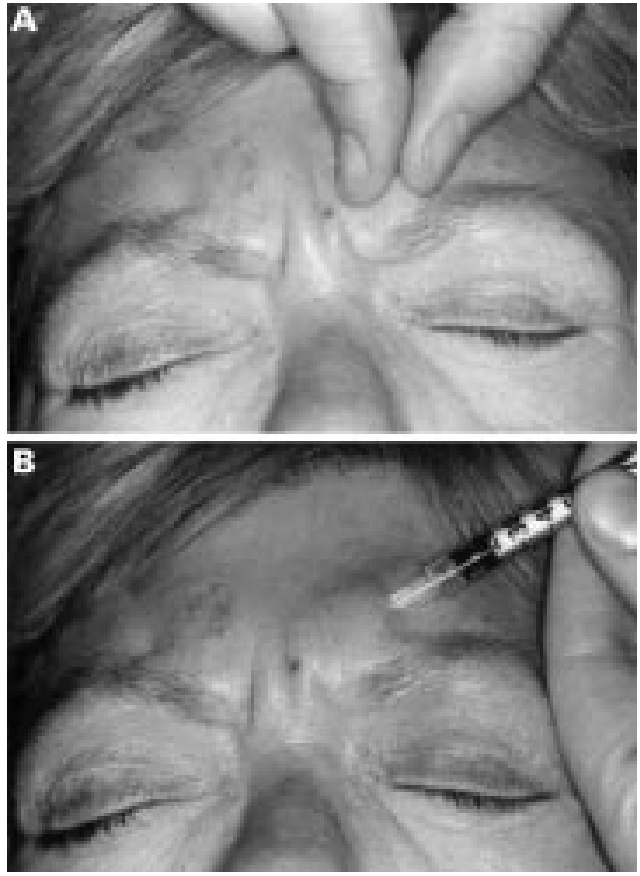

Figure 2 (A) Corrugator supercilii is easily palpated by asking the patient to contract these muscles. (B) Botulinum toxin $A$ is injected diagonally through the thick glabellar skin into the bulk of the muscle. The needle is directed medial and inferiorly. The muscle must be relaxed during the injection to reduce discomfort.

The procerus is a small fasciculate muscle continuous with the medial side of frontalis. It originates from the aponeurosis of the transverse nasalis, periosteum of the nasal bones, and perichondrium of the upper lateral cartilages. It inserts into the glabellar skin. It has similar actions to corrugator supercilii in that it helps to reduce the glare of bright sunlight by drawing the medial end of the eyebrow downwards. Procerus is also an elevator of the nose, to shorten it. ${ }^{9} 1112$

In thyroid eye disease the upper lid retraction, photophobia, and ocular discomfort act as a stimulus for contraction of these accessory muscles of eyelid closure (protraction).

\section{Methods}

Patients with thyroid eye disease who had clinically apparent overaction of corrugator supercilii plus or minus procerus muscles were identified by ophthalmologists in the thyroid and lid clinics at Moorfields Eye Hospital. The ophthalmologists assessed the eyebrow and eyelid contour and height as part of the normal thyroid eye disease assessment. Where constant glabellar furrowing from obviously active corrugator supercilii muscles was identified as a problem, the patient was invited to take part in the study. The study had full ethics committee approval and each patient gave informed consent.

All patients had inactive thyroid eye disease and were euthyroid at the time of injection. All patients had a history of chronic upper lid retraction of 2-10 years' duration (mean 4.3 years) and had noticeable vertical glabellar furrowing plus or minus a horizontal glabellar hyperfunction line, giving them a thyroid frown.

The injections were given as an outpatient procedure with the patient sitting upright in an examination chair. The glabellar region of the forehead was lightly cleaned with an alcohol wipe (Steret, Greton Prebbles Ltd). The glabellar muscles were first identified by asking the patient to contract them, then the injection made deep to the thick glabellar skin, at a point lateral to the glabellar lines (not into the lines), into the relaxed muscle. Botulinum toxin $\mathrm{A}$ (Dysport), $0.2 \mathrm{ml}$ (40 units), was injected into each corrugator muscle using a fine diabetic syringe with 27 gauge needle (Fig 2). Botulinum toxin A, $0.05 \mathrm{ml}$ (10 units), was injected into the procerus muscle from the mid glabellar region, vertically down towards the nasal bridge in selected patients.

The effect of a single injection of botulinum toxin A was assessed clinically and by questionnaire. Clinical and photographic documentation was made of the glabella appearance before and at regular interval after botulinum toxin $\mathrm{A}$ 

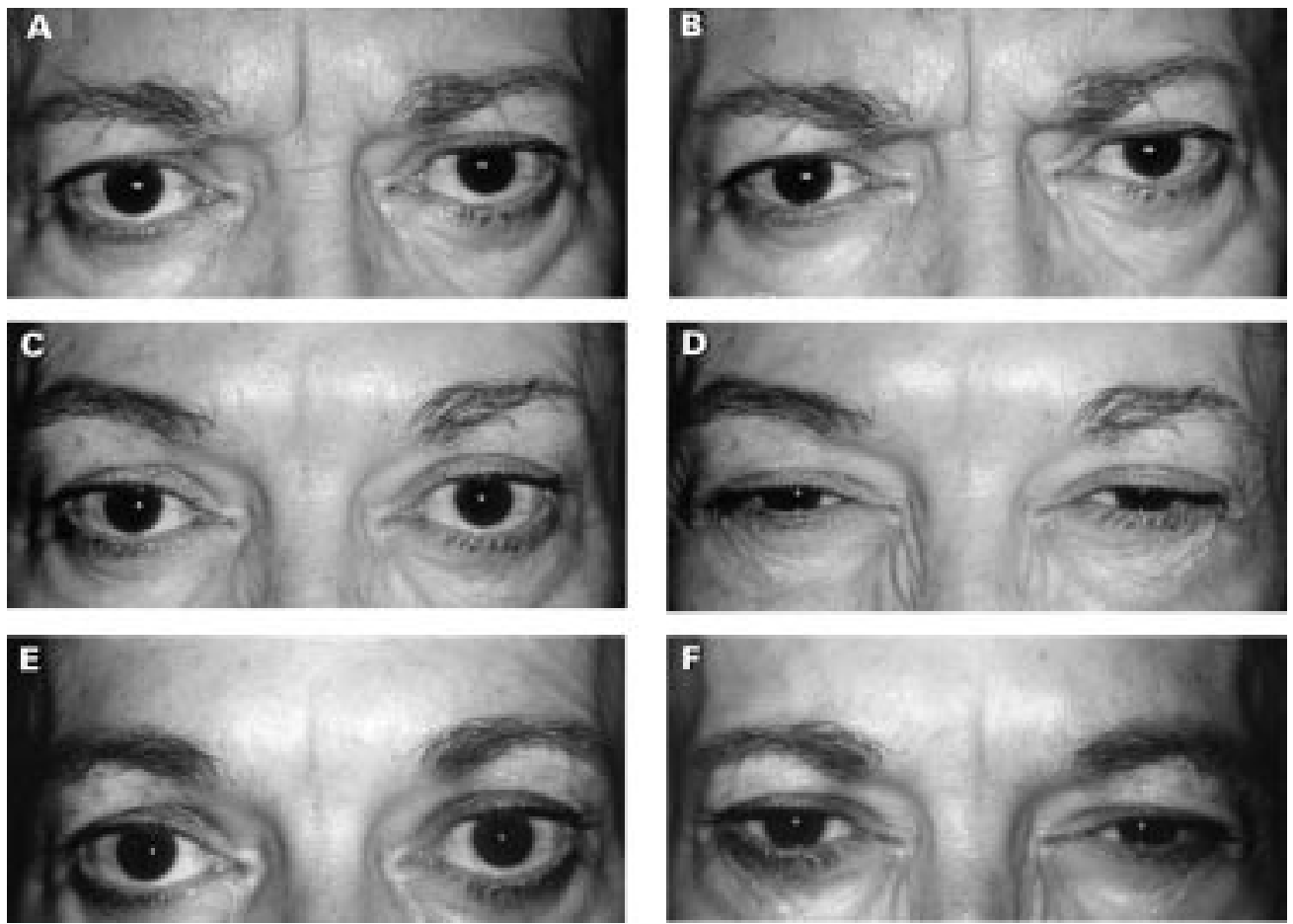

Figure 3 This 67 year old female thyroid patient had bilateral upper eyelid retraction for 5 years. Two years after bilateral Henderson's procedures, she still had prominent glabellar lines and bunching of the medial end of the eyebrows. She received botulinum toxin A injections into both corrugator supercilii and procerus muscles. After treatment she could not contract her glabellar muscles even with the greatest effort - the glabellar region remained smooth while the palpebral apertures narrowed (orbicularis oculi) and lateral nasal folds formed (nasalis) from increased facial nerve activity. These photographs show the pretreatment, early post-treatment (2 weeks) and longer term post-treatment (4 months) effects. Glabellar region: (A) Pretreatment. Relaxed. (B) Pretreatment. Actively contracting. (C) Two weeks after treatment. Relaxed. (D) Two weeks after treatment. Actively trying to contract. (E) Four months after treatment. Relaxed. (F) Four months after treatment. Actively trying to contract.

chemodenervation. Clinical measurements included the upper margin reflex distance.

The questionnaire included a grading of the discomfort from the injections, an estimate of the rapidity of onset of the effect, an estimate of the effect of treatment on their appearance and a report of side effects. Patients were also asked to estimate the glabellar muscle function as a percentage of normal, at each postoperative visit. Patients were asked whether they wished to continue with repeated toxin injections after the study was completed.

\section{Results}

Fourteen patients ( 13 female and 1 male), aged 39-76 years (mean 52, median 58), were entered into the study. The corrugator supercilii muscle alone was injected in nine patients and with procerus in five patients.

The timing of injections in relation to upper eyelid retraction surgery was 1 month before surgery $(n=3)$, at the time of surgery $(n=3)$, and 2-24 months (mean 7 months) after surgery $(n=8)$.

Most patients found the injections tolerable; six patients $(43 \%)$ reported no discomfort, four patients $(29 \%)$ experienced mild discomfort, three patients $(21 \%)$ tolerable discomfort. One patient $(7 \%)$ found the injections intolerable. The injections into corrugator were generally more tolerable than those into procerus.

The onset of glabellar muscle paralysis was within a week. Eleven patients $(79 \%)$ estimated they had complete paralysis at 1 week, while two (14\%) estimated $90 \%$ paralysis and one $(7 \%)$ estimated only $75 \%$ paralysis.

All patients noticed flattening of the vertical glabellar lines (Fig 3). The deep established furrows were not completely abolished (Fig 4). Patients described subjective improvement of glabellar appearance as follows: "nice and smooth, grooves less deep, feels stiff and numb, feels like a void, eyebrows lifted, forehead has more open look, funny feeling, cannot use it, its taken the nasty creases away, flat forehead, can't frown properly, feels more comfortable".

There were few immediate side effects from the injections. One patient $(7 \%)$ had slight ptosis for 6-8 weeks; this patient had inadvertently received twice the protocol dose. No patients in the study group reported diplopia. One patient noticed mild puffiness on the bridge of the nose after botulinum toxin A injection into procerus, which settled within 3 weeks and was attributed to a deep bruise. One patient had noticeable lateral nasal folds after a procerus injection, which settled (Fig 3).

The corrugator supercilii and procerus paralysis gradually wore off over 4-6 months (mean 4.5). At 3 months after injection, patients' estimation of return of function ranged from $20 \%$ to $90 \%$ (mean $42 \%$ ). Full return of protractor function occurred by 6 months. Many patients commented that even after full return of protractor function, they continued to keep these muscles relaxed for several weeks, even though they could contract them voluntarily. 

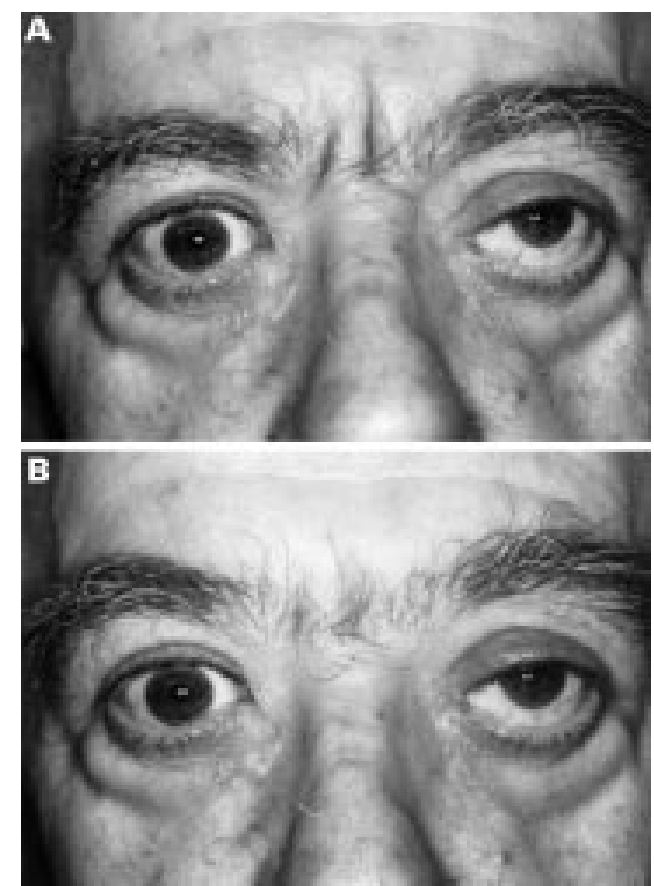

Figure 4 This 62 year old male thyroid patient had bilateral marked upper eyelid retraction for several years. Four months following bilateral levator recession he still had mild right upper eyelid retraction with marked glabellar furrows and altered eyebrow contour with bunched medial eyebrow ptosis. He received botulinum toxin $A$ into the corrugator muscles but not procerus. After treatment the glabellar region was smoother but the established furrows were still visible. There was improvement of eyebrow contour by elevation and lateral displacement of the medial eyebrow. (A) Pretreatment. Relaxed. (B) One week after treatment. Relaxed.

Of those patients who had levator recessions before glabellar chemodenervation $(n=8)$, five had inadequate surgery with residual upper eyelid retraction - with an upper margin reflex distance exceeding $4 \mathrm{~mm}$. The mean upper eyelid retraction, measured by upper margin reflex distance for this group was $3.50 \mathrm{~mm}$ (range $2.0-6.5 \mathrm{~mm}$ ).

Those patients who had glabella chemodenervation 1 month before or at the time of levator surgery had no residual upper lid retraction 4 months after surgery when the mean upper margin reflex distance was $2.4 \mathrm{~mm}$ (range $1.5-4 \mathrm{~mm}$ ). In particular, the patients who had successful correction of upper lid retraction did well in the long term, since the stimulus to glabellar muscle overaction was diminished. Within the time course of this study, 3/5 patients with residual upper eyelid retraction required further injections. One patient, whose upper lids remained severely retracted despite levator recessions (upper MRD 4.5 and 6.5), strongly requested continuing injections while awaiting repeat upper eyelid surgery. Two other patients with deep glabellar lines and mild residual upper lid retraction (upper MRD 5 and 4, 4 and 3) stated they would benefit from repeat injections. Documented follow up ranged from 4-12 months (mean 6 months) after injection, therefore the effect of treatment had not fully worn off in a number of patients and they could not judge whether they needed a further injection. The remaining patients who had successful upper eyelid surgery did not require further injections.

\section{Discussion}

All patients in this study reported a beneficial effect from botulinum treatment of their corrugator supercilii muscles. Patients with thyroid eye disease are very aware of how their thyroid facies differs from their former appearance. Our attention was first drawn to the problem of corrugator overaction by patients in the clinic describing their appearance as angry, cross looking, or scowling. Thyroid eye disease associated upper eyelid retraction as a cause of glabella frown lines has not been highlighted previously in the literature nor specifically treated.

Botulinum toxin $\mathrm{A}$ is a neurotoxin used to paralyse facial and eye muscles and has been used extensively by ophthalmologists for the treatment of strabismus, ${ }^{14}{ }^{15}$ benign essential blepharospasm, ${ }^{216-23}$ and corneal protection (protective ptosis). ${ }^{24}$ It has been used less frequently for the treatment of involutional entropion. $^{225-27}$ Ophthalmologists, neurologists, and otolaryngologists have used botulinum toxin $\mathrm{A}$ in the treatment of hemifacial spasm, ${ }^{16} 20232829$ other craniocervical muscle spasms, ${ }^{30}$ post-Bell's palsy facial synkinesis, ${ }^{31}$ and laryngeal dystonia. ${ }^{29}$ The paralysis is reversible and hence repeat focal injections are usually required to maintain the effect long term. The beneficial effect of botulinum toxin A on hyperfunctional facial lines was noted in the treatment of patients with blepharospasm and hemifacial spasm. ${ }^{2-5}$ Its aesthetic use has been promoted by oculoplastic, otolaryngology, and plastic surgeons. ${ }^{4-8}$ The corrugator supercilii muscles are easy to find and it is not necessary to locate them with electromyography, as has been suggested. ${ }^{6}$ They can readily be identified when the patient is asked to frown. Active contraction of corrugator supercilii bunches up the muscles which are then palpated. The injection is given obliquely through the thick glabellar skin into the superficial fascia layer or muscle, at the point of its maximum muscular contraction, and not into the actual skin lines as in previous studies. ${ }^{8}$

The procerus muscle is more difficult to palpate and its location is estimated from the position of the horizontal glabellar line. This muscle was only injected when there was a noticeable horizontal glabellar furrow in addition to vertical furrows. The discomfort associated with a procerus injection was worse than with the corrugator muscle injections and can spread inferiorly to the other muscles of the nose. It may be advantageous to limit the botulinum toxin A treatment to corrugator supercilii in the first instance and if there still is procerus overaction, inject this muscle subsequently. It is likely that enough botulinum toxin will diffuse into the bridge of the nose from the corrugator injection to the procerus muscle, as noticed in patients who had not received procerus injections.

Botulinum toxin A chemodenervation of the glabellar muscles produces an effective temporary paralysis (chemical rhytidectomy) with 
few complications. The patient with reversible partial ptosis had received double the normal dose-that is, 80 units to each corrugator, instead of 40 units, in error. She also had particularly lax tissue with a brow ptosis and hypermobile eyebrows which contributed to the tracking of botulinum toxin from the eyebrow to the levator muscle. Reversible ptosis is a recognisable side effect and has been recorded in $13.4 \%$ (range $0-52.5 \%$ ) of treatments with botulinum toxin A for blepharospasm ${ }^{30}$ and $0 \%$ to $9 \%$ for hyperkinetic facial lines. ${ }^{68}$

Diplopia has been reported in $2.1 \%$ $(0-17.2 \%)^{30}$ of treatments for blepharospasm, affecting either the superior rectus, inferior oblique, and inferior rectus muscles. There is a higher incidence of diplopia with increasing dose of toxin. ${ }^{19}$ Reversible diplopia has also been noted in $1 / 22(4.5 \%)$ of entropion patients treated with botulinum toxin, attributed to the spread of toxin to the inferior oblique muscle. ${ }^{29}$ Theoretically, botulinum toxin could also track back to the superior oblique, but this was not observed clinically in this study, nor has been reported following treatment of hemifacial and blepharospasm.

No patients in this small study developed post-treatment diplopia from orbital contamination by botulinum toxin. Fortunately, when side effects do occur from spread of botulinum toxin to adjacent tissues, they usually wear off within 1-6 weeks, while the target muscle remains paralysed for a longer period.

The best results of botulinum toxin treatment of hyperfunctional lines has been in the age group 30-50 years, where the tissue is not excessively lax. ${ }^{6}$ It would be prudent to either avoid treating elderly patients with particularly thin connective tissue or use lower doses of botulinum toxin, to reduce the risk of tissue spread causing side effects. In addition, glabellar lines in the elderly are invariably more established and deeper, hence more difficult to treat.

The beneficial effect of botulinum toxin was both flattening of the glabellar region and an improvement of eyebrow contour, particularly the position of the medial eyebrow. The medial brow is an area of specialised sliding superficial muscles (musculoaponeurotic structures) ${ }^{11}$ with a fat pad beneath the eyebrow within the deep galea aponeurotica. ${ }^{10}$ This fat pad extends into the upper eyelid as the areolar posterior orbicularis fascia. The brow fat pad attaches to the supraorbital ridge medially and centrally but there are no firm attachments laterally. In involutional brow ptosis, the lateral brow often becomes lower before the medial brow. However, in patients with thyroid eye disease with corrugator supercilii and procerus overaction, the brow is often lower medially, since these muscles assist the medial part of the orbital orbicularis oculi to depress the medial eyebrow. Botulinum toxin chemodenervation of corrugator supercilii and procerus will paralyse the adjacent orbicularis fibres, allowing the medial end of the eyebrow to lift and separate. Fibres from frontalis, the brow elevator muscle, also interdigitate with orbicular oculi at the medial end of the brow and should be similarly paralysed by botulinum toxin, causing a medial brow ptosis, but this effect was not noted in this study; presumably because the frontalis muscle is a larger stronger muscle.

The maximum effect of botulinum toxin $\mathrm{A}$ chemodenervation occurred between 5 and 10 days after injection, after which there was a slow recovery phase during which the patients experienced benefit. The beneficial effect of botulinum toxin A on glabellar hyperfunctional lines has been reported to last from 4 to 6 months. ${ }^{568}$ The patients in this study noted a similar prolonged benefit. This study was designed primarily to examine the effect of a single injection of botulinum toxin $\mathrm{A}$ into the glabellar muscles and did not examine the effect of repeated injections, although each patient was asked their opinion whether they needed another injection. In principle, where there is persistent upper eyelid retraction, the protractors will contract to try and reduce the palpebral apertures, thus reducing the glare, photophobia and discomfort: it is these patients who will require repeated injections. If there are already deeply established glabellar lines, these cannot be fully abolished with botulinum toxin nor after eyelid retractor surgery (Fig 4).

The prolonged benefit of botulinum toxin treatment of glabellar muscles may, in part, be due to temporary reconditioning of the voluntary and semivoluntary neural mechanisms which condition facial expression - that is, they learnt to inhibit habitual contraction of corrugator supercilii and procerus for a while. This allows the period between injections can therefore be as long as 6 months, rather than 3-4 months. However, in this study, we noted that once the stimulus to protraction from upper lid retraction has been reduced by successful levator recession surgery, the need to contract the glabellar muscles (frown) was reduced. Following this study, the decision to have repeat treatment depended mainly on whether there was still upper eyelid retraction acting as a stimulus to protraction. Since completion of this study, botulinum toxin A has become an established part of the management of patients in my practice who have thyroid eye disease with corrugator overaction and upper eyelid retraction. Its use is confined to those patients who are (i) awaiting upper lid surgery, (ii) in whom satisfactory lid lowering could not be obtained or surgery was declined and, rarely, (iii) where there has been satisfactory lid lowering but persistent glabellar lines.

\section{Summary}

The aesthetic effects of botulinum toxin A chemodenervation of corrugator supercilii and procerus muscles include reduction of medial brow ptosis with improvement of the eyebrow contour, and flattening of the glabellar region (apart from deep glabellar lines). This study shows that botulinum toxin $\mathrm{A}$ is a useful adjunctive treatment in the management and rehabilitation of patients with disfiguring thyroid eye disease, particularly where there is upper eyelid retraction and frown. The use of 
reversible chemical rhytidectomy should be considered as a therapeutic option in these patients. Once the upper eyelids are lowered, the stimulus for protraction is reduced and glabellar furrowing is less noticeable therefore the need for further injections is similarly reduced.

Originally presented as a poster at the Oxford Ophthalmological Congress, July 199

I thank Mr Peter Fells, Mr Richard Collin, and Mr John Lee at Moorfields Eye Hospital.

Botulinum toxin A from Dysport, Porton Products Ltd, Berkshire. There was no commercial interest in the use of Dysport from Porton Products.

1 Pierard GE, Lapiere CM. The microanatomical basis of facial frown lines. Arch Ophthalmol 1989;125:1090-2.

2 Carruthers J, Stubbs HA. Botulinum toxin for benign essential blepharospasm, hemifacial spasm and age-relate lower eyelid entropion. Can f Neurol Sci 1987;14:42-5.

3 Patrinely JR, Whiting AS, Anderson RL. Local side effects of botulinum toxin injections. Facial dyskinesias. In: Jankovic J, Tolosa E, eds. Advances in neurology, Vol 49. New York: Raven Press, 1988:493-500.

4 Carruthers JDE, Carruthers JA. Treatment of glabellar frown lines with C botulinum-A exotoxin. F Dermatol Surg frown lines with C bo

5 Blitzer A, Mitchell FB, Keen MS, et al. Botulinum toxin for the treatment of hyperfunction lines of the face. Arch the treatment of hyperfunction lines of the

6 Keen M, Blitzer A, Avis J, et al. Botulinum toxin A for hyperkinetic facial lines: results of a double blind placebo controlled study. Plast Reconstr Surg 1994;94:94-9.

7 Guyuron B, Huddleston SW. Aesthetic indications for botulinum toxin injection. Plast Reconstr Surg 1994;93:913-18.

8 Foster JA, Barnhorst D, Papay F, et al. The use of botulinum A toxin to ameliorate facial kinetic frown lines. Ophthalmology 1996;103:618-22.

9 Warwick R, Williams P, eds. The fasciae and muscles of the head. In: Gray's anatomy 35th ed. London: Longman 1973: 496-8.

10 Lemke BN, Stasior OG. The anatomy of eyebrow ptosis. Arch Ophthalmol 1982;100:981-6.

11 Abramo AC. Anatomy of the forehead muscles: the basis for the videoendoscope approach in forehead rhytidoplasty. the videoendoscope approach in
Plast Reconstr Surg 1995;95:1170-7.

12 Letourneau A, Daniel RK. The superficial musculoaponeurotic system of the nose. Plast Reconstr Surg 1988;82:48-55.
13 Ramirez OM. The anchor subperiosteal forehead flap. Plast Reconstr Surg 1995;95:993-1003.

14 Scott AB. Botulinum toxin injection in eye muscles to correct strabismus. Trans Am Ophthalmol Soc 1981;79:73470.

15 Fitzsimmons R, Lee JP, Elston J. Treatment of sixth nerve palsy in adults with combined botulinum toxin chemoden ervation and surgery. Ophthalmology 1988;95:1535-42.

16 Frueh BR, Felt DP, Wojno TH, et al. Treatment of blepharospasm with botulinum toxin. A preliminary report. Arch Ophthalmol 1984;102:1464-8.

17 Scott AB, Kennedy RA, Stubbs HA. Botulinum A toxin injection as a treatment of blepharospasm. Arch Ophthalmol 1985;103:347-50.

18 Shorr N, Seiff SR, Kopelman J. The use of botulinum toxin in blepharospasm. Am f Ophthalmol 1985;99:542-6.

19 Frueh BR, Musch DC. Treatment of facial spasm with botulinum toxin. An interim report. Ophthalmology 1986; 93:917-23.

20 Elston JS. Long term results of treatment of idiopathic blepharospasm with botulinum toxin injection. $\mathrm{Br} F$ Ophthalmol 1987;71:664-8.

21 Borodic GE, Cozzolino D. Blepharospasm and its treatment, with emphasis on the use of botulinum toxin. Plast Reconstr Surg 1989;83:546-54.

22 Grandas F, Elston J, Quinn N, et al. Blepharospasm: a review of 264 patients. F Neurol, Neurosurg, Psychiatry 1988;51:767-72

23 Taylor JDN, Kraft SP, Kazdan MS, et al. Treatment of blepharospasm and hemifacial spasm with botulinum A toxin: a Canadian multicentre study. Can $f$ Ophthalmol 1991;26:133-8

24 Adams GG, Kirkness CM, Lee JP. Botulinum toxin A induced protective ptosis. Eye 1987;1:603-8.

25 Lingua RW. Sequelae of botulinum toxin injection. $A m$ f Ophthalmol 1985;100:305-7.

26 Clarke JR, Spalton DJ. Treatment of senile entropion with botulinum toxin. Br $\mathcal{F}$ Ophthalmol 1988;72:361-2.

27 Hoh HB, Steel D, Potts MJ, et al. The use of botulinum toxin for lower lid entropion. Orbit 1995;14:131-6.

28 Gonnering RS. Treatment of hemifacial spasm with botulinum A toxin: results and rationale. Ophthal Plast Reconstr Surg 1986;2:143-6.

29 Brin MF, Fahn S, Moskowitz CB, et al. Localised injections of botulinum toxin for the treatment of focal dystonia and hemifacial spasm. Adv Neurol 1988;50:599-608.

30 Dutton JJ. Botulinum-A toxin in the treatment of craniocervical muscle spasms: short- and long-term, local and vical muscle spasms: short- and long-term,

31 Clark RP, Berris CE. Botulinum toxin: a treatment for facial asymmetry caused by facial nerve paralysis. Plast Reconstr Surg 1989;84:353-5. 